\title{
A comparison of ultra-endurance cyclists in a qualifying ultra-cycling race for Paris-Brest-Paris and Race Across America-Swiss cycling marathon
}

Knechtle, Beat ; Wirth, Andrea ; Knechtle, Patrizia ; Rüst, Christoph Alexander ; Rosemann, Thomas

\begin{abstract}
Ultra-endurance events test the adaptation of human physiology to extreme physical and mental demands, high levels of training, motivation, and physical conditioning among participants. To understand basic differences among participants according to the severity of the race, participants in qualifying events for two ultra-endurance cycling races, differing in length and intensity, were compared on measures of anthropometry, training, and support. One race was four times longer, required supporting teams, and racers typically had little sleep, which should lead to the qualifiers being substantially more highly trained than those from the shorter race. The qualifiers in the longer race had greater intensity in training while the qualifiers in the shorter race relied more on training volume. Different strategies and types of training reflected the different demands of the races. Future studies should evaluate personality and motivational differences in ultra-endurance events and between these athletes and athletes in other sports.
\end{abstract}

DOI: https://doi.org/10.2466/05.PMS.114.1.96-110

Posted at the Zurich Open Repository and Archive, University of Zurich

ZORA URL: https://doi.org/10.5167/uzh-64231

Journal Article

Accepted Version

Originally published at:

Knechtle, Beat; Wirth, Andrea; Knechtle, Patrizia; Rüst, Christoph Alexander; Rosemann, Thomas (2012). A comparison of ultra-endurance cyclists in a qualifying ultra-cycling race for Paris-Brest-Paris and Race Across America-Swiss cycling marathon. Perceptual and Motor Skills, 114(1):96-110.

DOI: https://doi.org/10.2466/05.PMS.114.1.96-110 


\title{
A COMPARISON OF ULTRA-ENDURANCE CYCLISTS IN A QUALIFYING ULTRA-CYCLING RACE FOR Paris-Brest-Paris AND FOR THE Race Across America - THE Swiss Cycling Marathon ${ }^{1,2}$
}

\author{
Beat Knechtle, Andrea Wirth, Patrizia Knechtle \\ Gesundheitszentrum St. Gallen \\ Switzerland \\ Christoph Alexander Rüst, Thomas Rosemann \\ Institute of General Practice and for Health Services Research \\ University of Zurich \\ Switzerland
}

\footnotetext{
${ }^{1}$ Address correspondence to Beat Knechtle, M.D., Facharzt FMH für Allgemeinmedizin, Gesundheitszentrum St. Gallen, Vadianstrasse 26, 9001 St. Gallen, Switzerland or e-mail (beat.knechtle@hispeed.ch).

${ }^{2}$ The authors thank the crew of the Swiss Cycling Marathon for their generous support and the athletes for their promptitude in enabling the collection of data before the race. Mary Miller from Stockton-on-Tees, Cleveland in England, helped with translation.
} 
Summary. - Ultra-endurance events test the adaptation of human physiology to extreme physical and mental demands, high levels of training, motivation, and physical conditioning among participants. To understand basic differences among participants according to the severity of the race, participants in qualifying events for two ultra-endurance cycling races, differing in length and intensity, were compared on measures of anthropometry, training and support. Both qualifying races took place within the annual Swiss Cycling Marathon. One race was $600 \mathrm{~km}$ long with $4,700 \mathrm{~m}$ of altitude gain ( $n=54$ participants), and provided qualification for the longest nonstop ultra-cycling race in Europe, Paris-Brest-Paris (PBP) which is $1,200 \mathrm{~km}$ long with $10,000 \mathrm{~m}$ of altitude. The other was $720 \mathrm{~km}$ with $5,580 \mathrm{~m}$ of altitude gain ( $n=30$ participants) and provided qualification for the longest nonstop ultracycling race in the USA, the Race Across America (RAAM) which is 5,000 km long with $30,000 \mathrm{~m}$ of altitude gain. Thus, the latter is four times longer, requires supporting teams, and racers typically have little sleep, which should lead to the qualifiers being substantially more highly trained than those from shorter races. The association between variables with race time was investigated using bi- and multivariate analysis. As expected, the 30 RAAM-qualifiers were younger, had lower body fat, lower skin-fold thicknesses, cycled more kilometres per year, showed a higher maximal cycling distance during training, a higher weekly cycling distance and trained for more hours a week in more training units with greater distance and a greater speed per unit than the 54 PBP-qualifiers. Interestingly, in multivariate regressions, anthropometric variables (e.g., skin-fold thickness, body fat) and support during the race were not related to race time for either group of cyclists. During the race, the PBP-qualifiers rode alone and without their own support crews providing nutrition and spare parts, in contrast to the RAAM-qualifiers. No RAAM-qualifier slept during the race whereas 31 of the 54 PBPqualifiers $(57 \%)$ slept for about two hours. Multiple linear regression analysis found that speed in cycling during training was related to race time in RAAM-qualifiers while mean duration per training unit was associated with race time in the PBP-qualifiers. To summarize, the RAAM-qualifiers had greater intensity in training while the PBP-qualifiers relied more on training volume. Different strategies and types of training reflected the different demands of the races. Future studies should evaluate personality and motivational differences in ultraendurance events and between these athletes and athletes in other sports. 
In recent years, several studies investigating ultra-endurance cyclists have been published, including both case reports and field studies. Their findings include differences among cyclists and changes in many variables related to the extreme race distances and training regimens, such as in body mass (Bircher, Enggist, Jehle, \& Knechtle, 2006; Knechtle, Enggist, \& Jehle, 2005; Knechtle, Wirth, Knechtle, \& Rosemann, 2009a), intensity and energy turnover (Bircher, et al., 2006; Francescato \& Di Prampero, 2002; Knechtle, et al., 2005; Reher, Hellemans, Rolleston, Rush, Miller, 2009; Wirnitzer \& Kornexl, 2008), and nutrition (Ebert, Martin, Stephens, McDonald, \& Withers, 2007; Martin, Martin, Collier, \& Burke, 2002). Other studies have tested associations between race performance measures and anthropometric and training variables (Knechtle \& Rosemann, 2009; Knechtle, Knechtle, Rosemann, 2009b; Knechtle, Wirth, Knechtle, \& Rosemann, 2009c).

In ultra-cycling, the most famous races for recreational ultra-endurance cyclists are the Race Across America (RAAM) in the USA (www.raceacrossamerica.org) and the ParisBrest-Paris (PBP) in Europe (www.paris-brest-paris.org). The Race Across America is also the longest non-stop ultra-cycling race all over the world. Although both races are ultracycling marathons, they have considerable differences: The Race Across America takes place every year; Paris-Brest-Paris occurs only every four years. Race Across America-cyclists have to cover $\sim 5,000 \mathrm{~km}$ with approximately $30,000 \mathrm{~m}$ of altitude, depending upon the course, whereas Paris-Brest-Paris is a nonstop ultra-cycling marathon over $\sim 1,200 \mathrm{~km}$ including $\sim 10,000 \mathrm{~m}$ of altitude. There are important differences between the two races in the use of support. In the Race Across America, athletes must be followed by a support crew of two cars providing equipment and nutrition during the whole race. One car is behind the cyclist to protect him from traffic while the other car is used for orientation and providing nutrition. In Paris-Brest-Paris, competitors can be followed by a support car providing equipment and nutrition. Drafting is forbidden in the Race Across America but is allowed in Paris-Brest-Paris. In the Race Across America, official finishers must complete the whole 
distance within 12 days; in the Paris-Brest-Paris, however, the athletes can choose to finish within 90 hours, 84 hours or 80 hours. For both races, the athletes have to qualify in order to take part. For Paris-Brest-Paris, cyclists must complete qualifying races over $200 \mathrm{~km}, 300 \mathrm{~km}$, $400 \mathrm{~km}$, and $600 \mathrm{~km}$, respectively. They have only to complete these qualifiers within the time limit set by the organizers. For the Race Across America, cyclists have to qualify in an ultra-cycling race over $720 \mathrm{~km}$ (444 miles) and must finish within $115 \%$ of the winners 'race time in order to qualify. The latter criterion should lead to greater competitiveness, as the outcome of qualifying is dependent on the cyclist's performance relative to the winner, rather than a set time. In the Swiss Cycling Marathon, qualifying races for both Paris-Brest-Parisqualifiers and Race Across America-qualifiers were offered.

To date, we do not know whether support during an ultra-endurance cycling race is related to race outcome. Having a support team should indicate that the cyclist has greater commitment, organization, and competitiveness, so it is expected that having a support team will be associated with faster race times in both groups. The purpose of this study was to investigate the association between training, anthropometry and support in cyclists participating in two races that are qualifying the cyclists for races of different intensity, length, and distance. It was assumed that anthropometry, training and nutrition, as well as factors such as equipment and support would be associated with race performance. Due to the much greater difficulty of the RAAM race, it was assumed that cyclists attempting to qualify for it would train harder and thus be superior to the PBP-qualifiers across all training variables, as well as having the effects of this increased training manifested in lower body fat and smaller skin-fold thicknesses. Since Paris-Brest-Paris-qualifiers only have to complete the qualifier within a time limit and Race Across America-qualifiers must finish within $115 \%$ of the winners 'time, it was assumed there would be differences in both cycling speed and support during the race. Specifically, it was hypothesized that the Race Across Americaqualifiers would ride faster during the qualifying race and rely more upon their own support 
crews compared to Paris-Brest-Paris-qualifiers. Finally, due to the longer race and more stringent qualifying standards for the Race Across America, it was hypothesized that the finishing rate would be lower than for the Paris-Brest-Paris race. 


\section{METHOD}

The Swiss Cycling Marathon takes place every year and offers different distances in order to qualify for Paris-Brest-Paris (600 km-qualifier race) or Race Across America (720 km-qualifier race). Data were collected in the 2007 and 2009 events, when both, the qualifier for Paris-Brest-Paris as well as the qualifier for the Race Across America were held. The organizer of the Swiss Cycling Marathon contacted all race participants three months before the start of the race and provided information about the planned investigation. Interested athletes contacted the investigator by e-mail and were provided with study documentation.

\section{Participants}

A total of 67 male participants out of 198 starters from the Paris-Brest-Paris-qualifier were interested in the study, and a total of 49 male participants out of 108 starters from the Race Across America-qualifier. All participants gave their informed written consent; the study was approved by the Ethical Committee of Canton St. Gallen, St. Gallen, Switzerland. In the Paris-Brest-Paris-qualifier, 54 participants with $45.2(S D=7.9)$ years of age, 78.9 $(S D=8.4) \mathrm{kg}$ of body mass, $1.81(S D=0.08) \mathrm{m}$ of body height and $24.2(S D=2.4) \mathrm{kg} / \mathrm{m}^{2}$ Body Mass Index finished the race within the time limit. A total of 30 participants with 41.4 $(S D=6.1)$ years of age, $75.0(S D=7.9) \mathrm{kg}$ of body mass, $1.79(S D=0.05) \mathrm{m}$ of body height and a Body Mass Index of $23.4(S D=1.6) \mathrm{kg} / \mathrm{m} 2$ finished in the Race Across America-qualifier.

\section{The Race}

The Swiss Cycling Marathon takes place at the end of June/start of July. In the $600 \mathrm{~km}$ race, the athletes pass ten check points and must cover a total altitude of $\sim 4,700$ metres. In the $720 \mathrm{~km}$ race, they must first complete the $600 \mathrm{~km}$ course of the race and then have to add an additional loop of $120 \mathrm{~km}$. Therefore, they have to pass 11 checkpoints and to cover $\sim 5,580$ 
metres of altitude in total. The $600 \mathrm{~km}$ loop starts from the outskirts of Berne (Switzerland) leading over the border to Germany, then following Lake Constance into the Alps of Eastern Switzerland and back to Berne. The organizer offered nutrition at each checkpoint such as hypotonic sports drinks, soup, sandwiches, fruits, energy bars and muesli. Weather conditions are extremely important in races this long and demanding. Because the athletes are already being heavily stressed by the distances, the addition of cold, wet, or hot conditions can drastically alter performance. Conditions were comparable in both years. In 2007 the weather in Berne was temperate and sunny at $22{ }^{\circ} \mathrm{Celsius}$ during the day and $11^{\circ} \mathrm{Celsius}$ in the night. In the 2009 race, the temperature rose to $25^{\circ} \mathrm{Celsius}$ and dropped to $9{ }^{\circ} \mathrm{Celsius}$. In both years there was no rain.

\section{Pre-Race Measurements and Calculations}

The participants kept a comprehensive training diary recording their training units in cycling, showing the distance $(\mathrm{km})$, duration $(\mathrm{h})$ and speed $(\mathrm{km} / \mathrm{h})$ for each training session for three months up to the start of the race after informed written consent. Before the start of the race, body mass, body height, the length of the arm and the leg, the circumferences of limbs and the thicknesses of eight skin-folds were measured. With this data, Body Mass Index, the sum of skin-folds, percent body fat, fat mass and fat-free mass, were calculated using an anthropometric method. Body mass was measured using a commercial scale (Beurer BF 15, Beurer, Ulm, Germany) to the nearest $0.1 \mathrm{~kg}$. Body height was determined using a stadiometer to the nearest $1.0 \mathrm{~cm}$. The circumferences and the lengths of the limbs were measured on the right side of the body, using a non-elastic tape measure $(\mathrm{cm})(\mathrm{KaWe} \mathrm{CE}$, Kirchner und Welhelm, Germany). The length of the arm was measured from acromion to the tip of the third finger to the nearest $0.1 \mathrm{~cm}$; the length of the leg from trochanter major to the malleolus lateralis to the nearest $0.1 \mathrm{~cm}$. The circumference of the upper arm was measured in the middle of the upper arm (between acromion and olecranon) to the nearest $0.1 \mathrm{~cm}$; the 
circumference of the thigh was taken at the level where the skin-fold thickness of thigh was measured (20 $\mathrm{cm}$ above the upper margin of the patella) and the circumference of the calf was measured at the maximum circumference of the calf. The skin-fold data were obtained using a skin-fold calliper (GPM-Hautfaltenmessgerät, Siber \& Hegner, Zurich, Switzerland) and recorded to the nearest $0.2 \mathrm{~mm}$. The skin-fold measurements were taken on the right side of the body following ISAK standard once for all eight skin-folds (pectoralis, axillar, triceps, subscapular, abdomen, suprailiac, thigh, and calf) and then the procedure was repeated twice more by the same investigator; the mean of the three times was then used for the analyses. The timing of the taking of the skin-fold measurements was standardised to ensure reliability. According to Becque, Katch, and Moffat (1986), readings were performed $4 \mathrm{~s}$ after applying the calliper. One trained investigator took all the skin-fold measurements as inter-tester variability is a major source of error in skin-fold measurements. An intra-tester reliability check was conducted on 27 male athletes prior to testing. Intra-class correlation (ICC) within the two judges was excellent for all anatomical measurement sites, and various summary measurements of skin-fold thicknesses (ICC > .9). Agreement tended to be higher within measurers than between measurers but still reached excellent reliability (ICC $>.9$ ) for the summary measurements of skin-fold thicknesses (Knechtle, Joleska, Wirth, Knechtle, Rosemann, \& Senn, 2010). Fat mass and fat-free mass were estimated using the equations following Stewart and Hannan (2000) for male athletes: Fat mass (g) $=331.5 \mathrm{x}$ abdominal skin-fold thickness $+356.2 \mathrm{x}$ thigh skin-fold thickness $+111.9 \mathrm{x}$ body mass -9108 ; fat-free mass $(\mathrm{g})=888 \mathrm{x}$ body mass $-252 \mathrm{x}$ abdominal skin-fold thickness $-382 \mathrm{x}$ suprailiacal skinfold thickness $-335 \mathrm{x}$ thigh skin-fold thickness +9120 . Percent body fat was estimated using the equation of Ball, Altena, and Swan (2004) for males: Percent body fat $=0.465+0.180 \mathrm{x}$ $(7 \mathrm{SF})-0.0002406 \times(7 \mathrm{SF})^{2}+0.0661 \mathrm{x}$ age, where $7 \mathrm{SF}$ is the sum of skin-fold thickness of pectoralis, axillar, triceps, subscapular, abdomen, suprailiac, and thigh mean in mm and age is in years. Prior to the race, the weight of the race bike was determined to the nearest $0.1 \mathrm{~kg}$ 
without additional equipment. After the race, the participants were asked whether they had completed the race alone or with the help of a support crew, whether they followed the signposts set by the organizer or used GPS (Global Positioning System), whether they carried their own equipment to mend a flat tire or whether they had spare parts or a complete replacement bike with them in the support car, and whether they used the nutrition provided by the organizer at the check points or whether they used their own nutrition. In addition, they reported whether they had taken a break for sleep and, if so, stated the time invested in sleeping.

\section{Statistical Analyses}

Data are presented as means and standard deviations $(S D)$. Variables of anthropometry and training between qualifiers for Paris-Brest-Paris and Race Across America were compared using Mann-Whitney-Wilcoxon tests. Fisher's exact test was applied for categorical data to assess the association between variables during the race with total race time. Race time was also expressed as a percentage of the course record (22:24 h:min for the $720 \mathrm{~km}$ and 19:03 h:min for the $600 \mathrm{~km}$, respectively). Pearson correlations were calculated between variables of anthropometry, training and race support such as material and nutrition and total race time, in order to eliminate weakly associated variables in a subsequent multiple linear regression model. Variables with significant associations after bivariate analysis were entered into the multiple linear regression model. A multiple linear regression was assessed, with race time as the dependent variable, separately for qualifiers for Paris-Brest-Paris and Race Across America. A probability value of less than 0.05 was accepted as significant. 


\section{RESULTS}

\section{Performance of the Participants}

The percentage of successful finishers in the Race Across America-qualifiers was lower when compared to the Paris-Brest-Paris-qualifiers. Forty-nine participants started in the Race Across America-qualifier, 30 cyclists (61\%) finished within the time limit of 40 hours. Sixty-seven participants started in the Paris-Brest-Paris-qualifier, 54 cyclists (80\%) finished. Among the Race Across America-qualifiers, six finishers (20\%) qualified for the Race Across America since they finished within $115 \%$ of the winners 'race time. All 54 finishers in the Paris-Brest-Paris-qualifier arrived within the time limit and were therefore qualified for ParisBrest-Paris. The Race Across America-qualifiers completed the $720 \mathrm{~km}$ within 1,610 $(S D=148) \mathrm{min}$, the Paris-Brest-Paris-qualifiers finished the $600 \mathrm{~km}$ after 1,727 (SD=292) min. Despite the $120 \mathrm{~km}$ difference in distances, the total race time was not significantly different between the two groups ( $p>.05)$. Thus, the participants in the Race Across America-qualifier cycled at $27.0(S D=2.3) \mathrm{km} / \mathrm{h}$, significantly faster compared to the Paris-Brest-Paris-qualifiers riding with $21.4(S D=3.5) \mathrm{km} / \mathrm{h}(\mathrm{p}<.0001)$. The Race Across America-qualifiers finished within $119(S D=11) \%$ of the course record, while qualifiers for Paris-Brest-Paris finished within $151 \%(S D=25, \mathrm{p}<.0001)$.

\section{Anthropometric characteristics of the Participants}

The Race Across America-qualifiers were significantly younger, had lower body fat, a lower sum of total skin-fold thicknesses, and lower skin-fold thicknesses at the upper and lower body (see Table 1). In the Race Across America-qualifiers, none of the anthropometric variables were related to total race time after bivariate analysis. In the Paris-Brest-Parisqualifiers, age, body fat percentage, fat mass, sum of total skin-folds and upper body skinfolds were related to total race time after bivariate analysis. In the Paris-Brest-Paris-qualifiers, 
age was significantly and positively related to percent body fat $(r=.36, p=.009)$, but not to yearly cycling distance $(r=-.07)$, weekly cycling volume $(r=-.14)$, or mean cycling speed during training $(r=.10)(p>.05)$.

\section{Training characteristics of the Participants}

The Race Across America-qualifiers cycled more kilometres per year (see Table 2), reported a higher maximal cycling distance per training unit during training $(+22 \%)$, a higher weekly cycling distance and trained for more hours per week in more training units at a higher velocity compared to the Paris-Brest-Paris-qualifiers. Cycling speed in training was significantly and negatively related to race time for the Race Across America-qualifiers in the bivariate analysis. Training volume, as measured by mean cycling distance per training unit and mean duration of a training unit, was significantly and negatively associated with total race time in the Paris-Brest-Paris-finishers after bivariate analysis.

\section{Equipment and Support of the Participants}

Racing bikes were significantly lighter in the Race Across America-qualifiers compared to the Paris-Brest-Paris-qualifiers (see Table 3). The Paris-Brest-Paris-qualifiers rode significantly more frequently alone during the race. No Race Across America-qualifier slept during the race whereas 31 Paris-Brest-Paris-finishers (57\%) slept for about two hours on average. In the bivariate analysis, racing with their own support crew and using their own nutrition was related with total race time in the Race Across America-qualifiers. In the ParisBrest-Paris-qualifiers, time invested in sleeping was associated with total race time (see Table 4). 


\section{Multiple Linear Regression Analysis}

When all significant variables after bivariate analysis were entered into a regression model, speed in cycling during training was significantly and negatively $(r=-.36, p<.05)$ related to race time in the Race Across America-qualifiers (see Table 5). For the Paris-BrestParis-qualifiers, mean duration per training was significantly and negatively $(r=-.31, p<.05)$ associated with total race time (see Table 6). 


\section{DISCUSSION}

The purpose of this study was to investigate the differences in both the anthropometry and the training in two groups of cyclists engaged in two different races within the Swiss Cycling Marathon, one $600 \mathrm{~km}$ in length and the other $720 \mathrm{~km}$ in length, as they attempted to qualify for ultra-endurance races of even greater lengths and intensities, Paris-Brest-Paris and the Race Across America, respectively. Additionally, the associations between training, anthropometry and support during the races in both groups of attempted qualifiers were evaluated and compared. Since the cyclists in the Race Across America-qualifier have to finish the qualifier race within $115 \%$ of the winners' time, only $20 \%$ of the successful finishers were qualified for the Race Across America in contrast to the cyclists in the ParisBrest-Paris-qualifier, where all successful finishers became qualified since they had only to finish the qualifier race within the time limit set by the race director. As hypothesized, due to the much longer and more rigorous race they were attempting to qualify for, the Race Across America-qualifiers cycled significantly faster than their Paris-Brest-Paris-counterparts, so much so that although the former travelled $120 \mathrm{~km}$ more than the latter, their overall race times were no different. The Race Across America-qualifiers invested more time in training, completed a higher training volume and trained at a higher cycling speed. Accordingly, they cycled faster during the race. As simple as this relationship between greater training and better performance is, another recent study of 28 male ultra-cyclists in a $600 \mathrm{~km}$ ultra-cycling race found that training volume was unrelated to race performance (Knechtle, With, Knechtle, \& Rosemann, 2009d). This may be due to the present sample actually training for a much more demanding race shortly in the future. For the present Race Across America-sample of 54 cyclists, the mean cycling distance per training unit and the mean duration per training unit were related to overall race time in the bivariate analysis, but only the mean duration per training unit was significant in the multiple linear regression analysis. 
The association of anthropometric characteristics with overall race time was also investigated because in prior studies of ultra-endurance athletes, anthropometric variables such as a lower body mass (Knechtle, Duff, Welzel, \& Kohler, 2009), a lower Body Mass Index (Hoffman, 2008), a lower body fat (Knechtle, et al., 2009d) and a lower upper arm circumference (Knechtle, Knechtle, Schulze, \& Kohler, 2007; Knechtle, et al., 2009a) were related to faster race times, presumably due to great training and increased conditioning. In ultra-cyclists, a lower body fat was associated with a faster race time (Knechtle, et al., 2009d), but not skin-fold thicknesses (Knechtle \& Rosemann, 2009; Knechtle, et al., 2009b). An interesting finding was that no anthropometric variables were related to overall race time after bivariate analysis in the Race Across America-qualifiers, but body fat and the sum of total skin-fold thicknesses were significantly and positively associated with overall race time in the Paris-Brest-Paris-qualifiers. In both groups, fat-free mass showed no association with overall race time. Regarding the multiple linear regression analysis, anthropometric variables were no longer significant in both groups and training variables remained significant. This confirms recent findings that training as indexed by overall body fat is important for performance in cyclists (Schumacher, Mroz, Mueller, Schmid, \& Ruecker, 2006). Anthropometric characteristics seem to be of no importance for a fast overall race time in ultra-endurance cyclists when controlled for covariates.

A second hypothesis was that the Race Across America-qualifiers would rely more upon their own support crews compared to the Paris-Brest-Paris-qualifiers because this is required in the race for which they are qualifying. In the Race Across America, the riders must have a support crew with two cars. In the Paris-Brest-Paris, the athletes can be supported by a car, but are not obliged to be followed by a support crew. Of the 30 Race Across America-qualifiers, 25 cyclists $(84 \%)$ were followed by a support crew whereas only 13 Paris-Brest-Paris-qualifiers (24\%) were supported. Riding with a support crew was significantly and positively associated with overall race time in the Race Across America- 
qualifiers, but not in the Paris-Brest-Paris-qualifiers. We assume that the Race Across America-qualifiers were highly motivated and interpret the findings that having a support team indicates greater preparation, more organization, and more commitment to the outcome of the qualifier. Regarding the equipment, the Race Across America-qualifiers had a highly significantly lighter racing bike compared to the Paris-Brest-Paris-qualifiers. However, the weight of the bike was not related to overall race time in the bivariate analysis. In both groups, no difference regarding the use of GPS for orientation and the use of spare parts in case of a defect was found. Since in both groups only one athlete had to give up due to problems with his bike, technical problems with the bike seem not to limit race performance in ultra-endurance cycling.

An interesting finding was that the Paris-Brest-Paris-qualifiers were highly significantly more likely to rely upon the nutrition provided by the organizer compared to the Race Across America-qualifiers. The Race Across America-qualifiers, on the other hand, were using significantly more of their own nutrition provided by the support crew. Twentythree Race Across America-qualifiers (77\%) had their own support crew; however, only nine Paris-Brest-Paris-qualifiers (17\%) were followed by a support crew. In the bivariate analysis, racing with a support crew and using their own nutrition were significantly related with total race time in the Race Across America-qualifiers, but remained no longer significant when controlled with co-variates in the multiple linear regression analysis. Nonetheless, the Race Across America-qualifiers seemed more motivated and better prepared for the race than the Paris-Brest-Paris-qualifiers.

The Race Across America-qualifiers did not take any breaks during the race, whereas $57 \%$ of the Paris-Brest-Paris-qualifiers took a break for sleep. This break obviously did not enhance performance (Knechtle, Knechtle, Wirth, Rosemann, Rüst, \& Lepers, 2012) since sleep time and total race time were significantly and positively related; overall riding time increased due to the breaks. This finding can easily be explained since the Race Across 
America-qualifiers have to finish within $115 \%$ of the winner's time in order to qualify for the Race Across America, so they are extremely motivated to continue racing and not lose any time to sleep because it means losing time to other cyclists who do not sleep but continue to ride. The Paris-Brest-Paris-qualifiers, however, only need to finish within the time limit set by the race director to get the qualification for Paris-Brest-Paris, so sleep can be factored into their overall time and not reduce their chances at qualifying. A recent study at the Race Across America investigated the strategy in one athlete (Schumacher, Ahlgrim, Prettin, \& Pottgiesser, 2011). The athlete used a strategy with regular sleeping breaks and the authors concluded that in contrast to conventional racing strategies for the Race across America, which aimed at minimizing sleep and maximizing ride time, their case demonstrated that by emphasizing regular recovery and sleep, such alternative strategy might lead to an equally successful race result, but clearly this strategy has not been widely accepted among the athletes. We assume that experienced ultra-endurance athletes have enough previous experience in order to develop and follow their self-selected pacing strategy (Herbst, Knechtle, Lopez, Andonie, Fraire, Kohler, et al., 2011).

\section{Limitations of the present study}

We collected data in two different years where weather conditions were not absolutely identical. In a recent study it has been shown that finish times in ultra-marathons were affected by warm weather (Wegelin \& Hoffman, 2011). The specific types of nutrients and energy intake were not determined; specific nutrients certainly can affect the race outcome (Knechtle, Knechtle, Schulze, \& Kohler, 2008; Peters, 2003; Rehrer, et al., 2009). 


\section{CONCLUSIONS}

Although variables of both anthropometry and support were related to total race time in the bivariate analysis, they were no longer significant in the multiple linear regression analysis when controlled with covariates. The Race Across America-qualifiers seem to rely more upon intensity in training whilst the Paris-Brest-Paris-qualifiers rely more upon volume in training. We assume that ultra-endurance athletes develop their own strategy in training and racing in order to successfully finish an ultra-endurance race. However, motivational aspirations to enter such a qualifier, on the one hand, and intending to start in a considerably longer race, on the other hand, might presumably play a large role in the outcome of the race. In future studies, the aspect of motivation to compete in such ultra-endurance races should be further investigated. 


\section{REFERENCES}

Ball, S.D., Altena, T.S., and Swan, P.D. (2004) Comparison of anthropometry to DXA: a new prediction equation for men. European Journal of Clinical Nutrition, 58, 1525-1531.

Becque, M. D., Katch, V. L., \& Moffatt, R. J. (1986) Time course of skin-plus-fat compression in males and females. Human Biology, 58, 33-42.

Bircher, S., Enggist, A., Jehle, T., \& Knechtle B. (2006) Effects of an extreme endurance race on energy balance and body composition - a case study. Journal of Sports Science and Medicine, 5, 154-162.

Ebert, T. R., Martin, D. T., Stephens, B., McDonald, W., \& Withers, R. T. (2007) Fluid and food intake during professional men's and women's road-cycling tours. International Journal of Sports Physiology and Performance, 2, 58-71.

Francescato, M. P., \& Di Prampero, P. E. (2002) Energy expenditure during an ultraendurance cycling race. The Journal of Sports Medicine and Physical Fitness, 42, 1-7.

Herbst, L., Knechtle, B., Lopez, C. L., Andonie, J. L., Fraire, O. S., Kohler, G., Rüst, C. A., \& Rosemann, T. (2011) Pacing strategy and change in body composition during a Deca Iron Triathlon. Chinese Journal of Physiology, 54, 255-263.

Hoffman, M. D. (2008) Anthropometric characteristics of ultramarathoners. International Journal of Sports Medicine, 29, 808-811.

Knechtle, B., Duff, B., Welzel, U., \& Kohler, G. (2009a) Body mass and circumference of upper arm are associated with race performance in ultraendurance runners in a multistage race--the Isarrun 2006. Research Quarterly for Exercise and Sport, 80, 262268.

Knechtle, B., Enggist, A., \& Jehle, T. (2005) Energy turnover at the Race Across AMerica (RAAM) - a case report. International Journal of Sports Medicine, 26, 499-503. 
Knechtle, B., Joleska, I., Wirth, A., Knechtle, P., Rosemann, T., \& Senn, O. (2010) Intra- and inter-judge reliabilities in measuring the skin-fold thicknesses of ultra runners under field conditions. Perceptual and Motor Skills, 111, 105-106.

Knechtle, B., Knechtle, P., \& Rosemann, T. (2009b) No association between skinfold thicknesses and race performance in male ultra-endurance cyclists in a $600 \mathrm{~km}$ ultracycling marathon. Human Movement, 10, 91-95.

Knechtle, B., Knechtle, P., Schulze, I., \& Kohler, G. (2007) Upper arm circumference is associated with race performance in ultra-endurance runners. British Journal of Sports Medicine, 42, 295-299.

Knechtle, B., Knechtle, P., Schulze, I., \& Kohler, G. (2008) Vitamins, minerals and race performance in ultra-endurance runners--Deutschlandlauf 2006. Asia Pacific Journal of Clinical Nutrition, 17, 194-198.

Knechtle, B., Knechtle, P., Wirth, A., Rosemann, T., Rüst, C. A., \& Lepers, R. (2012) No improvement in race performance by naps in male ultra-endurance cyclists in a 600km ultra-cycling race. Chinese Journal of Physiology, in press

Knechtle, B., \& Rosemann, T. (2009) No correlation of skin-fold thickness with race performance in male recreational mountain bike ultra-marathoners. Medicina Sportiva, $13,152-156$

Knechtle, B., Wirth, A., Knechtle, P., \& Rosemann, T. (2009c) An ultra-cycling race leads to no decrease in skeletal muscle mass. International Journal of Sports Medicine, 30, 163-167.

Knechtle, B., Wirth, A., Knechtle, P., \& Rosemann, T. (2009d) Moderate association of anthropometry, but not training volume, with race performance in male ultraendurance cyclists. Research Quarterly for Exercise and Sport, 80, 563-568. 
Martin, M. K., Martin, D. T., Collier, G. R., \& Burke, L. M. (2002) Voluntary food intake by elite female cyclists during training and racing: influence of daily energy expenditure and body composition. International Journal of Sport Nutrition and Exercise Metabolism, 12, 249-267.

Peters, E. M. (2003) Nutritional aspects in ultra-endurance exercise. Current Opinion in Clinical Nutrition and Metabolic Care, 6, 427-434.

Rehrer, N. J., Hellemans, I. J., Rolleston, A. K., Rush, E., \& Miller, B. F. (2010) Energy intake and expenditure during a 6-day cycling stage race. Scandinavian Journal of Medicine and Science in Sports, 20, 609-618.

Schumacher, Y. O., Ahlgrim, C., Prettin, S., \& Pottgiesser, T. (2011) Physiology, power output and racing strategy of a Race across America (RAAM) Finisher. Medicine and Science in Sports and Exercise, 43, 885-889.

Schumacher, Y. O., Mroz, R., Mueller, P., Schmid, A., \& Ruecker, G. (2006) Success in elite cycling: A prospective and retrospective analysis of race results. Journal of Sports Sciences, 24, 1149-1156.

Stewart, A. D., \& Hannan, W. J. (2000) Prediction of fat mass and fat-free mass in male athletes using dual X-ray absorbtiometry as the reference method. Journal of Sports Sciences, 18, 263-274.

Wegelin, J. A., \& Hoffman, M. D. (2011) Variables associated with odds of finishing and finish time in a 161-km ultramarathon. European Journal of Applied Physiology, 111, 145-153.

Wirnitzer, K. C., \& Kornexl, E. (2008) Exercise intensity during an 8-day mountain bike marathon race. European Journal of Applied Physiology, 104, 999-1005. 


\section{TABLE 1}

Comparison of Anthropometric Characteristics of the Finishers and Pearson Correlations with Total Race Time

\begin{tabular}{|c|c|c|c|c|c|c|c|}
\hline \multirow[b]{2}{*}{ Variables } & \multicolumn{3}{|c|}{$\begin{array}{l}\text { Race Across America-qualifiers } \\
(n=30)\end{array}$} & \multicolumn{4}{|c|}{$\begin{array}{l}\text { Paris-Brest-Paris-qualifiers } \\
(n=54)\end{array}$} \\
\hline & Mean & $S D$ & Pearson $r$ & Mean & $S D$ & Pearson $r$ & $p$ \\
\hline Age, yrs & $41.4 *$ & 6.1 & .14 & 45.2 & 7.9 & $.30, p=.0267$ & .03 \\
\hline Body height, $\mathrm{m}$ & 1.79 & 0.05 & .07 & 1.81 & 0.08 & .04 & \\
\hline Body mass, $\mathrm{kg}$ & 75.0 & 7.9 & .08 & 78.9 & 8.4 & .17 & \\
\hline Body mass index, $\mathrm{kg} / \mathrm{m}^{2}$ & 23.4 & 1.6 & .06 & 24.2 & 2.4 & .16 & \\
\hline Length of leg, $\mathrm{cm}$ & 88.3 & 3.2 & -.20 & 88.5 & 5.0 & -.02 & \\
\hline Length of arm, $\mathrm{cm}$ & 81.3 & 3.4 & -.19 & 81.1 & 4.5 & .08 & \\
\hline Circumference of upper arm, $\mathrm{cm}$ & 29.9 & 1.9 & .13 & 30.6 & 2.2 & -.03 & \\
\hline Circumference of thigh, $\mathrm{cm}$ & 56.0 & 2.7 & -.03 & 56.7 & 3.3 & -.20 & \\
\hline Circumference of calf, $\mathrm{cm}$ & 38.5 & 2.2 & .05 & 38.1 & 2.2 & -.11 & \\
\hline Percent body fat, $\%$ & $13.5 * *$ & 3.5 & .15 & 16.9 & 4.3 & $.43, p=.0017$ & .002 \\
\hline Fat mass, $\mathrm{kg}$ & $7.1 * *$ & 3.4 & .20 & 10.7 & 4.7 & $.34, p=.0115$ & .01 \\
\hline Fat-free mass, $\mathrm{kg}$ & 65.2 & 6.8 & .03 & 63.7 & 7.7 & -.09 & \\
\hline Sum of eight skin-folds, $\mathrm{mm}$ & $67.4 * *$ & 25.2 & .16 & 92.3 & 32.1 & $.40, p=.0039$ & .004 \\
\hline Sum of upper body skin-folds, mm & $52.6 * *$ & 21.4 & .17 & 72.6 & 26.2 & $.41, p=.0029$ & .003 \\
\hline Sum of lower body skin-folds, $\mathrm{mm}$ & $14.8 *$ & 5.7 & .18 & 19.7 & 8.7 & .23 & \\
\hline
\end{tabular}

Note.- $* p<.05, * * p<.01 . P$-value is inserted in case of a significant association 


\section{TABLE 2}

Comparison of Characteristics of Training and Prerace Experience for Finishers and Pearson Correlations with Total Race Time

\begin{tabular}{|c|c|c|c|c|c|c|c|c|}
\hline \multirow[b]{2}{*}{ Variables } & \multicolumn{4}{|c|}{$\begin{array}{l}\text { Race Across America-qualifiers } \\
(n=30)\end{array}$} & \multicolumn{4}{|c|}{$\begin{array}{l}\text { Paris-Brest-Paris-qualifiers } \\
(n=54)\end{array}$} \\
\hline & Mean & $S D$ & $r$ & $p$ & Mean & $S D$ & $r$ & $p$ \\
\hline Years as active cyclist, yrs & 10.8 & 5.7 & -.32 & & 14.2 & 10.0 & .02 & \\
\hline Mean kilometres covered in the year before race, $\mathrm{km}$ & $13,759 * *$ & 6,484 & -.36 & & 9,160 & 6,093 & -.25 & \\
\hline Number of training units in cycling per week, $n$ & $5.3 * *$ & 2.2 & -.15 & & 3.3 & 1.4 & -.05 & \\
\hline Minimal cycling distance per training unit, $\mathrm{km}$ & 46.3 & 19.5 & .06 & & 49.5 & 27.8 & -.30 & \\
\hline Maximal cycling distance per training unit, $\mathrm{km}$ & $279.3 * *$ & 86.3 & -.32 & & 216.2 & 68.2 & -.23 & \\
\hline Mean cycling distance per training unit, $\mathrm{km}$ & 99.3 & 24.8 & .22 & & 87.0 & 29.0 & -.36 & .02 \\
\hline Mean duration per training unit, min & 195.7 & 54.0 & .26 & & 196.1 & 81.1 & -.31 & .0449 \\
\hline Mean speed per training unit, $\mathrm{km} / \mathrm{h}$ & $29.3 * *$ & 1.7 & -.43 & .049 & 27.3 & 2.0 & -.07 & \\
\hline Mean weekly distance in cycling, $\mathrm{km}$ & $440.0 * *$ & 150.9 & -.17 & & 307.0 & 181.3 & -.12 & \\
\hline Mean weekly duration of training, $\mathrm{h}$ & $14.9 * *$ & 4.8 & -.14 & & 11.4 & 6.8 & -.18 & \\
\hline Number of finished qualifiers, $\mathrm{n}$ & $\begin{array}{c}2.7 \\
(n=9)\end{array}$ & 1.5 & .48 & & $\begin{array}{c}2.8 \\
(n=21)\end{array}$ & 2.1 & -.14 & \\
\hline
\end{tabular}

Note. $* p<.05, * * p<.01 . P$-value is inserted in case of a significant association 


\section{TABLE 3}

Comparison of Equipment, Support and Nutrition during the Race for Finishers

\begin{tabular}{lcc}
\hline Variables & $\begin{array}{c}\text { Race Across America-qualifiers } \\
(\boldsymbol{n}=\mathbf{3 0})\end{array}$ & $\begin{array}{c}\text { Paris-Brest-Paris-qualifiers } \\
(\boldsymbol{n}=\mathbf{5 4})\end{array}$ \\
\hline Weight of the race bike, kg & $7.7(0.8) * *$ & $9.1(1.3)$ \\
Racing alone & $5 * *$ & 41 \\
Racing with support crew & 25 & 13 \\
Racing following signpost set by organiser & 20 & 45 \\
Racing following GPS & 10 & 9 \\
Racing with tubes and pump & $7 * *$ & 45 \\
Racing with complete replacement bike & 23 & 9 \\
Racing using organiser's nutrition & $4 * *$ & 48 \\
Racing using own nutrition & 26 & 26 \\
Rest with sleep, min & 0 & $118(98)$ \\
& & $(n=31)$ \\
\hline
\end{tabular}

Note.- Values for bike weight and time for sleeping are given as mean (SD). ${ }^{* *} p<.01$. 


\section{TABLE 4}

Pearson Correlations of Equipment, Nutrition and Sleep with Total Race Time

\begin{tabular}{|c|c|c|c|c|}
\hline \multirow[b]{2}{*}{ Variable } & \multicolumn{2}{|c|}{$\begin{array}{c}\text { Race Across America-qualifiers } \\
\qquad(n=30)\end{array}$} & \multicolumn{2}{|c|}{$\begin{array}{c}\text { Paris-Brest-Paris-qualifiers } \\
(n=54)\end{array}$} \\
\hline & $r$ & $p$ & $r$ & $p$ \\
\hline Weight of the race bike & .07 & & .38 & \\
\hline Racing with support crew & .41 & .023 & -.17 & \\
\hline Racing following GPS & -.03 & & -.19 & \\
\hline Racing with replacement bike & .30 & & -.20 & \\
\hline Racing using own nutrition & .57 & .001 & -.16 & \\
\hline Sleep during the race & & & .50 & .004 \\
\hline
\end{tabular}

Note.- $P$-value is inserted in case of a significant association 


\section{TABLE 5}

Multivariate Analysis with Race Time as the Dependent Variable for the Race Across America-Qualifiers $(n=30)$

\begin{tabular}{llllll}
\hline & \multicolumn{2}{l}{ Unstandardized coefficients } & \multicolumn{2}{l}{$\begin{array}{l}\text { Standardized } \\
\text { Coefficients }\end{array}$} \\
\cline { 2 - 4 } Model & $\boldsymbol{B}$ & $\boldsymbol{S E}$ & $\boldsymbol{\beta}$ & $\boldsymbol{t}$ & $\boldsymbol{p}$-value \\
\hline Speed in cycling during training & -34.74 & 14.66 & -36.64 & -2.5 & .023 \\
Racing with support crew & -161.4 & 73.03 & -33.13 & -0.45 & .065 \\
Racing using own nutrition & -243.3 & 81.89 & -171.8 & -2.1 & .051 \\
\hline
\end{tabular}

Note. $R=0.658, R^{2}=0.433$, adjusted $R^{2}=0.333$, Standard error of estimate $=114.08, \mathrm{~F}_{\mathrm{df}}=4.32, p=.019$ 


\section{TABLE 6}

Multivariate Analysis with Race Time as the Dependent Variable for the Paris-Brest-Paris-Qualifiers ( $n=54)$

\begin{tabular}{|c|c|c|c|c|c|}
\hline \multirow[b]{2}{*}{ Model } & \multicolumn{2}{|c|}{ Unstandardized coefficients } & \multirow{2}{*}{$\begin{array}{l}\text { Standardized } \\
\text { Coefficients } \\
\beta\end{array}$} & \multirow[b]{2}{*}{$t$} & \multirow[b]{2}{*}{$p$-value } \\
\hline & $B$ & $S E$ & & & \\
\hline Mean duration per training unit & -6.09 & 2.05 & -.67 & -2.96 & .0066 \\
\hline Age & 4.02 & 11.87 & 0.91 & .339 & .741 \\
\hline Percent body fat & 65.52 & 117.34 & 1.15 & .558 & .588 \\
\hline Fat mass & -86.74 & 50.03 & -1.66 & -1.73 & .111 \\
\hline Sum of eight skin-folds & -11.52 & 19.28 & -1.57 & -.598 & .562 \\
\hline Sum of upper body skin-folds & 22.15 & 10.85 & 2.50 & 2.04 & .066 \\
\hline Mean cycling distance per training unit & -6.09 & 2.05 & -.672 & -2.96 & .130 \\
\hline Sleep during the race & .20 & .50 & .079 & .40 & .69 \\
\hline
\end{tabular}

Note. $R=0.856, R^{2}=0.732$, adjusted $R^{2}=0.562$, Standard error of estimate $=183.88, \mathrm{~F}_{\mathrm{df}}=4.303, p=.016$ 